Investigations

\title{
A Study of Prescribing Pattern of Drugs during Pregnancy and Lactation in the Secondary and Tertiary Care Hospitals of Bangladesh: A Cross Sectional Study
}

\author{
${ }^{1}$ Mohammad Mohasin Miah, ${ }^{2}$ Shakil Ahammad Mridha, ${ }^{2}$ Azad Md. Abu Rayhan and ${ }^{1}$ Afia Ferdous \\ ${ }^{1,2}$ Department of Pharmacy, Stamford University Bangladesh, 51 Siddeswari Road, Dhaka-1217, Bangladesh
}

Corresponding Author: Afia Ferdous

Department of Pharmacy, Stamford University

Bangladesh, 51 Siddeswari

Road, Dhaka-1217, Bangladesh

Phone: +88-01732-614841

Email: afia_sn004@yahoo.com

\begin{abstract}
Using of prescribed drugs during pregnancy and lactation requires special attention because of having risk of the mothers as well as the health and life of her unborn child. Medicinal abuse usually happens due to lack of abundant information on the use of drugs during pregnancy and lactation. The main objective of this study was to assess the information about the prevalence of the use of prescribed drugs among pregnant and lactating women in Bangladesh. This was a cross-sectional study and conducted at both the Rangpur Medical College Hospital (RMCH) and Institute of Child and Mother Health (ICMH) at Dhaka in Bangladesh. Medicinal prescription copies given to 500 pregnant women and 500 lactating mothers respectively at each hospital, who attended in the hospitals, were collected. While analyzing, it was found that on an average, per prescription of pregnancy and lactation contained 2.216 $\pm 0.019(\mathrm{P}=$ $0.50)$ and $1.199 \pm 0.014(\mathrm{P}=0.05)$ drugs respectively. The therapeutic classes and both US-FDA pregnancy and Thomas Hale lactation categories of the drugs prescribed to pregnant women and lactating mother were also analyzed. Anti-anemic drugs including iron preparations and vitamin and mineral supplements $(37.23 \%)$ were the most frequently prescribed medicines at both hospitals. Including vitamin and mineral supplements, 1098 women $(49.55 \%)$ received drugs from category A; 729 women (32.90\%) received drugs from category B; 309 women received drugs from category C (13.94\%); 80 women (3.61\%) received drugs from category D and no pregnant women received a drug from category $\mathrm{X}$ of the US-FDA risk classification system, whereas the highest number $(605,50.46 \%)$ of lactating mothers took drugs from $\mathrm{L}_{2}$ safe category of lactation and 355 (29.61\%) lactating mothers received anti-infective drugs from therapeutic classes during visiting at both hospitals. The study shows that the prescription of harmful drugs decreases during pregnancy and lactation in Bangladesh.
\end{abstract}

Keywords: Pregnancy, Lactation, Therapeutic Class, Prescription, US-FDA Category and Thomas Hale Lactation

\section{Introduction}

Pregnancy is a special physiological state where medication intake presents a challenge and a concern due to altered drug pharmacokinetics and drug crossing the placenta possibly causing harm to the fetus (Banhidy et al., 2005). Medication treatment in pregnancy can not be totally avoided since some pregnant women may have chronic pathological conditions that require continuous or interrupted treatment (e.g., asthma, epilepsy and hypertension) (Noha and Ahmed, 2014). Also during pregnancy new medical conditions can develop and old one can worsen (e.g., migraine, headache, hyperacidity, nausea, vomiting) requiring drug therapy (Deborah et al., 2005). However, before taking any drug (including overthe-counter drugs) or dietary supplements (including medicinal herbs), a pregnant woman should consult her health care practitioner. A health care practitioner may recommend that a woman take certain vitamins and minerals during pregnancy (Merck, 2007). Health care practitioners also consider of the benefits to the mother and the risk to the fetus while prescribing drugs during 
pregnancy (Rohra et al., 2008). It is not possible to avoid drugs during pregnancy, so women with certain chronic medical conditions such as epilepsy, diabetes, inflammatory bowel disease and asthma, the use of drugs is essential and benefits for mother and child may well outweigh the teratogenic risk of the drug. Other nonchronic diseases related or unrelated to the pregnancy may require medical treatment (Bakker et al., 2006).

The drugs prescribed to pregnant mothers for therapeutic purposes may cause serious structural and functional adverse effects in the developing child (Kacew, 1994). Reducing medication errors and improving patient safety are the important areas of discussion (Benjamin, 2003). It is essential to consider several factors before prescription of drugs during pregnancy. Such as, (a) Dose and duration of drug exposure is important. The larger the dose is more likely the effects. The longer the duration of drug exposure is greater chance of susceptible periods of organogenesis and developmental problem. (b) Timing of exposure is very crucial. Certain organ systems may have only limited period of susceptibility for damage. (c) Pathogenetic mechanism, teratogen produces their adverse effect by specific mechanism. (d) Host susceptibility, variability in the genetic factors related to mechanism of certain drugs. All drugs can affects the health of the mother and fetus, therefore any drugs should be administer with care during pregnancy (Pramanik and Saha, 2010).

Many mothers also require using drugs during breastfeeding. Almost all drugs transfer into breast milk and this may carry a risk to a breastfed infant. Factors such as the dose received via breast milk, pharmacokinetics and the effect of the drug in the infant need to be taken into consideration during prescribing (Sharon, 2001). Physicians also investigate the stage of the lactation, the age of the baby, the condition of the baby (particularly if preterm or ill) and the number of breastfeeds or amount of breast milk the infant receives (Denise, 2006). Most drugs likely to be prescribed to the breast feeding mother should have no effect on milk supply or on infant well-being (AAPCD, 2001). Most women who continue their drug use during pregnancy are unlikely to stop it during lactation. However, the study was conducted to appraise the patterns of drug prescriptions to pregnant women and lactating mother at secondary and tertiary care hospitals in Bangladesh.

\section{US-FDA Pregnancy Risk Factor Categories}

The USA Food and Drug Administration (FDA) classify the drugs for use in pregnancy using 5-letters system.

$\mathrm{A}=$ adequate Controlled studies in pregnant women fail to demonstrate a risk to the fetus. Very few drugs are seen in this category. B = "Best" No risk seen in animals, but no controlled trials in pregnant women. $\mathrm{C}=$ "Caution" "Adverse fetal effects in animals, no controlled trials in humans." Most drugs are category C. $\mathrm{D}=$ "Danger" "Evidence of human fetal risk should be reserved for life-threatening disease." $\mathrm{X}=$ strong evidence of fetal abnormality, No therapeutic indication in pregnancy. Teratogenic Drugs: "Most Teratogenic FDA-approved medications are in categories D or X, some drugs in C" (Briggs et al., 1994).

Prescribers should look the US-FDA risk categories when prescribes to pregnancy and lactation (Rose Ann L. Jankowski, 2013).

\section{Thomas Hale Lactation Risk Categories}

Thomas Hale categorizes lactation drugs using five letters.

$\mathrm{L}_{1}=$ "Safest" Scientific studies in breastfeeding women do not show any risks to their children. $\mathrm{L}_{2}=$ "Safe" A limited number of breastfeeding women have used this medicine and no increase in adverse effects in their children has been observed. $\mathrm{L}_{3}=$ "Fairly Safe" Scientific studies in breastfeeding women show only minor, non-life-threatening side effects in the child. $\mathrm{L}_{4}=$ "Possibly Risk" Scientific studies show that there is a potential risk for the child or milk production. $\mathrm{L}_{5}=$ "Canceled" Scientific studies in breastfeeding mothers show that it is dangerous for a child if his mother is using this medicine (Hale and Rowe, 2014).

\section{Methods}

We performed a cross-sectional study at two secondary and tertiary care hospitals in Bangladesh i.e. Rangpur Medical College Hospital (RMCH) and Institute of Child and Mother Health (ICMH) in which we investigated how accurately prescriptions reflected the actual use of medications during pregnancy and lactation. Rangpur medical college is situated in Rangpur, a northern part of Bangladesh, $330 \mathrm{~km}$ from Dhaka and $210 \mathrm{~km}$ from Rajshahi. ICMH is located at Matuali in Dhaka, adjacent to Dhaka-Chittagong highway. Rangpur Medical College Hospital (RMCH) was conveniently surveyed at the first phase from 16th April 2011 to 25th June 2011 and Institute of Child and Mother Health (ICMH) was surveyed from $1^{\text {st }}$ July 2011 to 15th September 2011. The sampling units were carefully selected so as to get the data that can maximally represent the prescribing trends in Bangladesh. Ethical clearance was obtained from the Ethical Review Committee of Stamford University Bangladesh. A written clearance was obtained from the Heads of the sampling units for obtaining data from their respective Hospitals. In order to prevent bias, the prescribers were kept unaware about the collection of prescriptions. From each hospital, copies of outpatient 
drug prescriptions given to 500 pregnant women and 500 lactating mothers respectively were collected.

This survey consisted of multiple choice questions. An English language survey was developed based on information drawn from relevant literatures pertaining to using of prescribed drugs during pregnancy and lactation in Bangladesh. Questionnaires for patient survey covered basic questions related to their illness, their trend to stick to doctor's prescription and related information (Table 1 and 2). Questionnaires were given only to the candidates whom were requested by the representatives and among the candidates who showed spontaneous interest to respond on the questions during the survey and then the questionnaires were filled up by the representatives. Also, recommended pathological tests of pregnancy (hCG), Complete Blood Count (CBC), blood typing and antibody screening, glucose fasting plasma, CUS fasting, Glucose 2 ABF plasma, CUS $2 \mathrm{~h}$ ABF, Thyroid Stimulating Hormone (TSH), hepatitis B screening, rubella immunity, urine culture and sensitivity, ultrasonography and Sexual Transmitted Diseases (STDs) were recorded from the latest laboratory investigation reports (Table 3). Problems of lactating mothers those were detected by the physicians on the basis of symptoms and diagnosis, were recorded (Table 4). All the completed questionnaires, however, were pooled together. Furthermore, both the brand names and generic names of prescribed drugs were documented and then the pharmacological classes of each drug prescribed to pregnant women and lactating mother were identified by the drug index of Bangladesh.

\section{Statistical Analysis}

Statistical analysis was expressed as mean \pm SEM and calculated using the software Graph Pad Quick Calcs: $t$ test calculator, USA. All the data were analyzed using Statistical Package for Social Sciences (SPSS) software (Version 22, IBM Corporation, USA). The statistically significant difference was considered at the level of $\mathrm{P}=0.05$ and 0.50 .

\section{Results}

All the pregnant women and lactating mother attending at the hospitals of two sampling units received a prescription containing at least one drug during their visits. As evident from Table 1, the maximum number of prescriptions was collected from women at both hospitals in their third trimester of pregnancy (44.0, $41.6 \%)$ followed by second $(37,35.4 \%)$ and first trimester $(19,23 \%)$, whereas breast feeding mothers who had a child of aging below 2 months received the highest number of prescriptions $(74.4,68.8 \%)$ from both $\mathrm{RMCH}$ and ICMH (Table 2). While analyzing the number of drugs prescribed to pregnant and lactating women, it was found out that on an average, at both centers each prescription of prescribers contained 2.216 \pm 0.019 and $1.199 \pm 0.014$ drugs respectively.

On reviewing pathological data, it was revealed that 2369 pathological tests were recommended to the women who were either pre-conceived or pregnant. Of all, 774 women $(32.67 \%)$ were recommended to test Ultrasonography, whilst $631(26.64 \%)$ recommendations were on glucose fasting plasma, CUS fasting, glucose 2 $\mathrm{h}$ ABF plasma, CUS $2 \mathrm{~h} \mathrm{ABF}$ and 583 (24.61\%) tests were on Complete Blood Count (CBC) and rest of the tests were on urine culture and sensitivity (147, 6.21\%), pregnancy test (hCG) $(104,4.39 \%)$, Thyroid Stimulating Hormone (TSH) $(82,3.46 \%)$, blood typing and antibody screening $(25,1.06 \%)$, Sexual Transmitted Diseases (STDs) (20, 0.84\%) and Hepatitis B screening (3, $0.13 \%$ ) (Table 3). Pathological data of breastfeeding mothers also showed that out of 219,139 (63.47\%) lactating mothers had the problem of milk production, whereas $45(20.55 \%)$ breastfeeding mothers who listed in this study, had been suffered from breast engorgement followed by sore nipples or breasts $(22,10.05 \%)$ and breast abscess 13 (5.94\%) (Table 4).

In addition, therapeutical classes of drugs prescribed to pregnant women were analyzed. As shown in Table 5, anti-anemic drugs including iron preparations and vitamin and mineral supplements $(37.23 \%)$ were the most frequently prescribed drugs followed by antiulcerants and anti-emetic $(22.38 \%)$, analgesics, antipyretic and anti-inflammatory (11.91\%), anti-infective $(6.0 \%)$, cardiovascular drugs including anti-coagulants (5.87\%), dermatological/antifungal $(4.02 \%)$, antidiabetic $(3.47 \%)$, hormonal drugs $(3.43 \%)$, antiasthmatic (2.93\%), anti-malarial/anti-protozoal (1.49\%) and CNS drugs including anti-epileptics (1.26\%).In contrast, including vitamin and mineral supplements, in total at both hospital, 1098 women $(49.55 \%)$ received a drug from category A; 729 women (32.90\%) received a drug from category B; 309 women received a drug from category C (13.94\%); 80 women $(3.61 \%)$ received a drug from category $\mathrm{D}$ and no women received a drug from category $\mathrm{X}$ of the US-FDA risk classification system in the 270 days before delivery (Table 6).

Figure 1 demonstrated that the physicians of ICMH centre prescribed more drugs to pregnant women from therapeutic classification of anti-anemic including vitamin and mineral supplements, anti-ulcerants and anti-emetic, analgesics, anti-pyretic, anti-inflammatory, CNS drugs including anti-epileptic, anti-asthmatic and hormonal drugs compared to the physicians of RMCH. Inversely, the physicians of RMCH during pregnancy gave more drugs from others therapeutic classification. Additionally, according to US-FDA category, it is seen that a little difference showed of prescribed drugs between two hospitals (Fig. 2). Neither hospital's physician prescribed a drug from category $\mathrm{X}$ of the USFDA risk classification. 


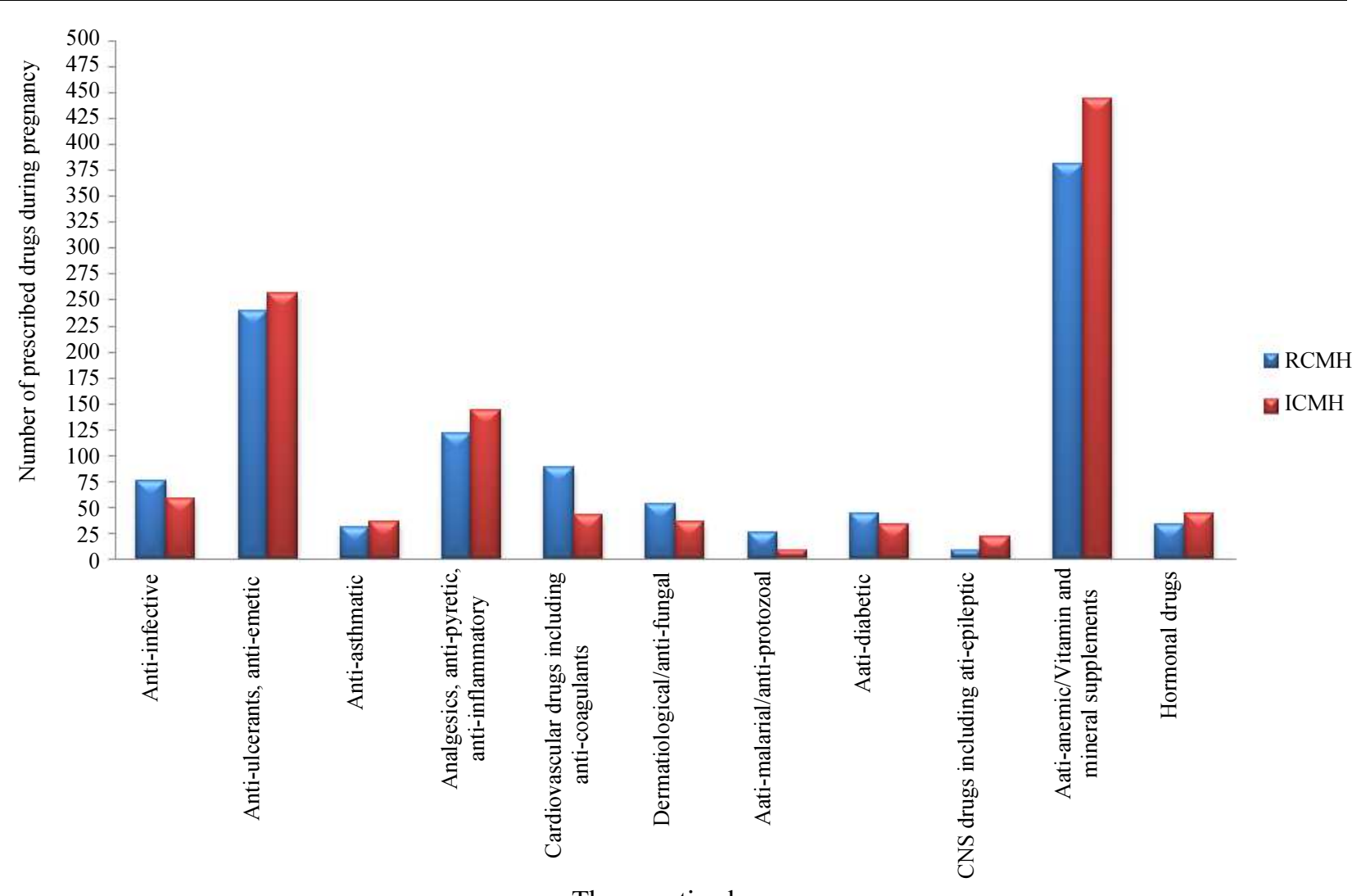

Fig. 1: Comparison of number of prescribed drugs between two hospitals according to therapeutic classes

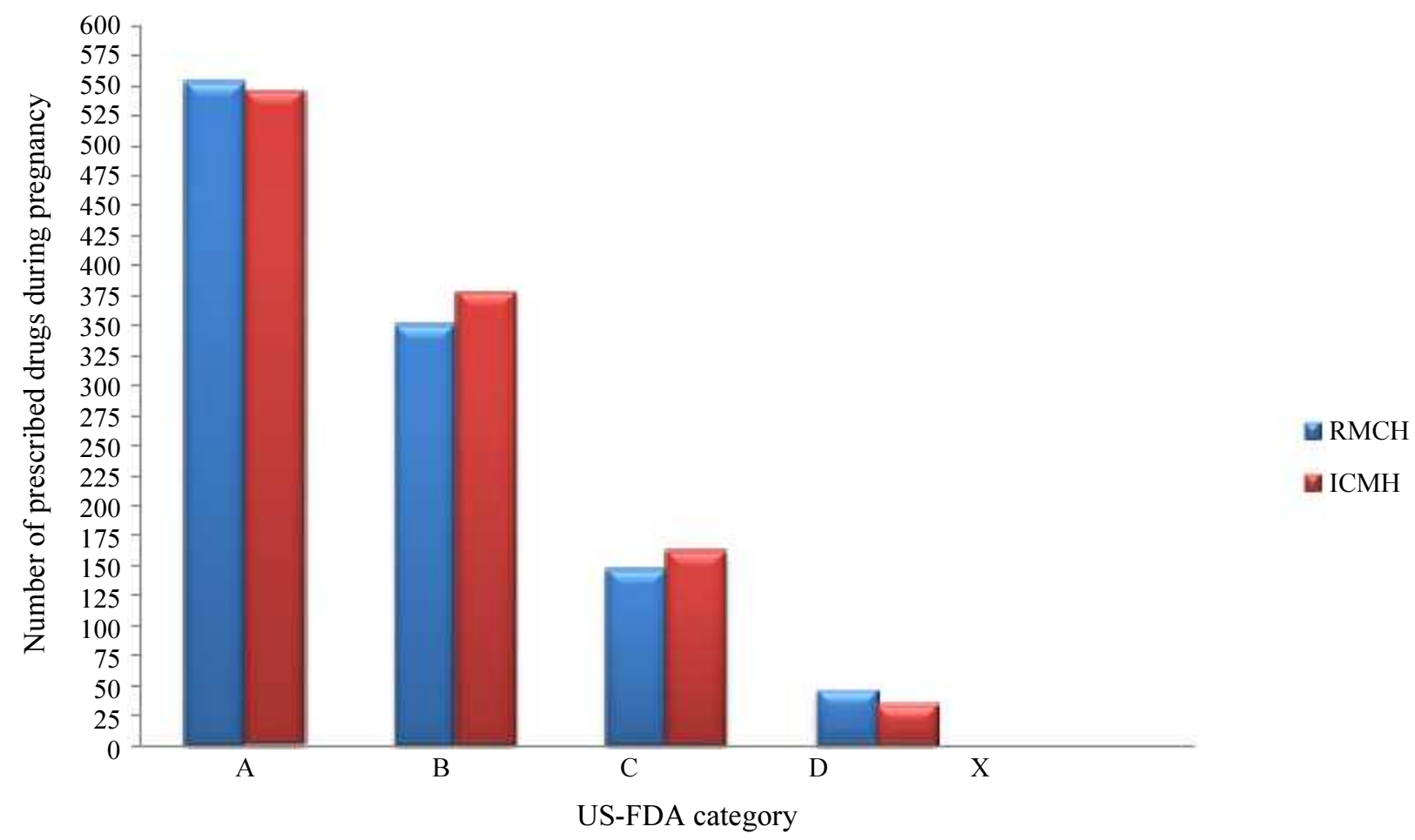

Fig. 2: Comparison of number of prescribed drugs between two hospitals according to US-FDA Category 
Table 1: Questionnaires based results of pregnant women' survey

\begin{tabular}{|c|c|c|c|c|c|c|}
\hline \multirow[b]{3}{*}{$\begin{array}{l}\text { Questionnaires for the } \\
\text { pregnant women }\end{array}$} & \multicolumn{6}{|c|}{ Number of surveyed patients at each hospital, $\mathrm{N}=500$} \\
\hline & \multicolumn{2}{|l|}{$\mathrm{RMCH}$} & \multicolumn{2}{|l|}{$\mathrm{ICMH}$} & \multirow[b]{2}{*}{$\begin{array}{l}\text { Total } \\
\text { response }\end{array}$} & \multirow[b]{2}{*}{$\begin{array}{l}\% \text { of tota } \\
\text { response }\end{array}$} \\
\hline & $\begin{array}{l}\text { No. of } \\
\text { response }\end{array}$ & $\begin{array}{l}\% \text { of } \\
\text { response }\end{array}$ & $\begin{array}{l}\text { No. of } \\
\text { response }\end{array}$ & $\begin{array}{l}\% \text { of } \\
\text { response }\end{array}$ & & \\
\hline \multicolumn{7}{|c|}{ Gestational period (Trimester) } \\
\hline First & 95 & 19.0 & 115 & 23.0 & 210 & 21.0 \\
\hline Second & 185 & 37.0 & 177 & 35.4 & 362 & 36.2 \\
\hline Third & 220 & 44.0 & 208 & 41.6 & 428 & 42.8 \\
\hline Total & 500 & 100.0 & 500 & 100.0 & 1000 & 100.0 \\
\hline \multicolumn{7}{|l|}{ Age of women (Years) } \\
\hline 14-less than 20 & 260 & 52.0 & 283 & 56.6 & 543 & 54.3 \\
\hline $21-25$ & 195 & 39.0 & 148 & 29.6 & 343 & 34.3 \\
\hline $26-30$ & 45 & 9.0 & 53 & 10.6 & 98 & 9.8 \\
\hline $31-35$ & 0 & 0.0 & 16 & 3.2 & 16 & 1.6 \\
\hline Above 35 & 0 & 0.0 & 0 & 0.0 & 0 & 0.0 \\
\hline Total & 500 & 100.0 & 500 & 100.0 & 1000 & 100.0 \\
\hline \multicolumn{7}{|l|}{ Occupation } \\
\hline House wife & 425 & 85.0 & 376 & 75.2 & 801 & 80.1 \\
\hline Private job & 60 & 12.0 & 69 & 13.8 & 129 & 12.9 \\
\hline Worker & 15 & 3.0 & 55 & 11.0 & 70 & 7.0 \\
\hline Total & 500 & 100.0 & 500 & 100.0 & 1000 & 100.0 \\
\hline \multicolumn{7}{|l|}{ Number of live birth } \\
\hline First time & 355 & 71.0 & 338 & 67.6 & 693 & 69.3 \\
\hline Second time & 115 & 23.0 & 126 & 25.2 & 241 & 24.1 \\
\hline Third time & 30 & 6.0 & 36 & 7.2 & 66 & 6.6 \\
\hline Fourth time & 0 & 0.0 & 0 & 0.0 & 0 & 0.0 \\
\hline Total & 500 & 100.0 & 500 & 100.0 & 1000 & 100.0 \\
\hline \multicolumn{7}{|c|}{ Family history of diabetes and hypertension } \\
\hline Yes & 143 & 28.6 & 118 & 23.6 & 261 & 26.1 \\
\hline No & 357 & 71.4 & 382 & 76.4 & 739 & 73.9 \\
\hline Total & 500 & 100.0 & 500 & 100.0 & 1000 & 100.0 \\
\hline \multicolumn{7}{|c|}{ Family history of epilepsy } \\
\hline Yes & 0 & 0.0 & 0 & 0.0 & 0 & 0.0 \\
\hline No & 500 & 100.0 & 500 & 100.0 & 1000 & 100.0 \\
\hline Total & 500 & 100.0 & 500 & 100.0 & 1000 & 100.0 \\
\hline \multicolumn{7}{|c|}{ Gestational complications } \\
\hline Hypertension & 0 & 0.0 & 0 & 0.0 & 0 & 0.0 \\
\hline Diabetes mellitus & 14 & 2.8 & 9 & 1.8 & 23 & 2.3 \\
\hline Epilepsy & 0 & 0.0 & 0 & 0.0 & 0 & 0.0 \\
\hline Others & 486 & 97.2 & 491 & 98.20 & 977 & 97.7 \\
\hline Total & 500 & 100 & 500 & 100.00 & 1000 & 100.0 \\
\hline \multicolumn{7}{|c|}{ Having any chronic disease before pregnancy } \\
\hline Yes & 0 & 0.0 & 0 & 0.0 & 0 & 0.0 \\
\hline No & 500 & 100.0 & 500 & 100.0 & 1000 & 100.0 \\
\hline Total & 500 & 100.0 & 500 & 100.0 & 1000 & 100.0 \\
\hline \multicolumn{7}{|l|}{$\mathrm{BP}$} \\
\hline $120 / 80$ & 120 & 24.0 & 133 & 26.6 & 253 & 25.3 \\
\hline Less than $120 / 80$ & 275 & 55.0 & 282 & 56.4 & 557 & 55.7 \\
\hline Above 120/80 & 105 & 21.0 & 85 & 17.0 & 190 & 19.0 \\
\hline Total & 500 & 100.0 & 500 & 100.0 & 1000 & 100.0 \\
\hline \multicolumn{7}{|l|}{ Blood glucose $(\mathrm{mmol} / \mathrm{L})$} \\
\hline Less than 7 & 486 & 97.2 & 491 & 98.2 & 977 & 97.7 \\
\hline Above 7 & 14 & 2.8 & 9 & 1.8 & 23 & 2.3 \\
\hline Total & 500 & 100.0 & 500 & 100 & 1000 & 100 \\
\hline \multicolumn{7}{|c|}{ Regular antenatal check up } \\
\hline Yes & 137 & 27.4 & 121 & 24.2 & 258 & 25.8 \\
\hline No & 363 & 72.60 & 379 & 75.8 & 742 & 74.2 \\
\hline Total & 500 & 100 & 500 & 100.0 & 1000 & 100.0 \\
\hline
\end{tabular}


Table 2: Questionnaires based results of lactating mothers' survey

\begin{tabular}{|c|c|c|c|c|c|c|}
\hline \multirow[b]{3}{*}{$\begin{array}{l}\text { Questionnaires for } \\
\text { the lactating patients }\end{array}$} & \multicolumn{6}{|c|}{ Number of surveyed patients at each hospital, $\mathrm{N}=500$} \\
\hline & \multicolumn{2}{|l|}{$\mathrm{RMCH}$} & \multicolumn{2}{|l|}{ ICMH } & \multirow[b]{2}{*}{$\begin{array}{l}\text { Total } \\
\text { response }\end{array}$} & \multirow[b]{2}{*}{$\begin{array}{l}\% \text { of total } \\
\text { response }\end{array}$} \\
\hline & $\begin{array}{l}\text { No. of } \\
\text { response }\end{array}$ & $\begin{array}{l}\% \text { of } \\
\text { response }\end{array}$ & $\begin{array}{l}\text { No. of } \\
\text { response }\end{array}$ & $\begin{array}{l}\% \text { of } \\
\text { response }\end{array}$ & & \\
\hline Yes & 500 & $100 \%$ & 500 & $100 \%$ & 1000 & $100 \%$ \\
\hline No & 0 & $0.0 \%$ & 0 & $0.0 \%$ & 0 & $0.0 \%$ \\
\hline Total & 500 & $100 \%$ & 500 & $100 \%$ & 1000 & $100 \%$ \\
\hline \multicolumn{7}{|l|}{ Age of child (Years) } \\
\hline Below 2 month & 372 & $74.4 \%$ & 344 & $68.8 \%$ & 716 & $71.6 \%$ \\
\hline Below 6 month & 97 & $19.4 \%$ & 109 & $21.8 \%$ & 206 & $20.6 \%$ \\
\hline More than 6 month & 31 & $6.2 \%$ & 47 & $9.4 \%$ & 78 & $7.8 \%$ \\
\hline Total & 500 & $100 \%$ & 500 & $100 \%$ & 1000 & $100 \%$ \\
\hline \multicolumn{7}{|l|}{ Mode of delivery } \\
\hline Normal & 352 & $70.4 \%$ & 337 & $67.4 \%$ & 689 & $68.9 \%$ \\
\hline Cesarean section & 148 & $29.6 \%$ & 163 & $32.6 \%$ & 311 & $31.1 \%$ \\
\hline Total & 500 & $100 \%$ & 500 & $100 \%$ & 1000 & $100 \%$ \\
\hline \multicolumn{7}{|l|}{ History of any birth defect } \\
\hline Yes & 0 & $0 \%$ & 0 & $0 \%$ & 0 & $0.0 \%$ \\
\hline No & 500 & $100 \%$ & 500 & $100 \%$ & 1000 & $100 \%$ \\
\hline Total & 500 & $100 \%$ & 500 & $100 \%$ & 1000 & $100 \%$ \\
\hline
\end{tabular}

Table 3: Recommendation of pathological tests to pregnant women on the basis of physical symptoms

\begin{tabular}{|c|c|c|c|c|c|c|c|c|c|}
\hline \multirow[b]{3}{*}{ Test name } & \multicolumn{8}{|c|}{ Gestational age (Trimester) } & \multirow[b]{3}{*}{$\begin{array}{l}\text { Total } \\
\mathrm{n}(\%)\end{array}$} \\
\hline & \multicolumn{4}{|l|}{$\mathrm{RMCH}$} & \multicolumn{4}{|l|}{ ICMH } & \\
\hline & $\begin{array}{l}\text { Pre-conception } \\
\mathrm{n}(\%)\end{array}$ & $\begin{array}{l}\text { First } \\
\mathrm{n}(\%)\end{array}$ & $\begin{array}{l}\text { Second } \\
\mathrm{n}(\%)\end{array}$ & $\begin{array}{l}\text { Third } \\
\mathrm{n}(\%)\end{array}$ & $\begin{array}{l}\text { Pre-conception } \\
\mathrm{n}(\%)\end{array}$ & $\begin{array}{l}\text { First } \\
\mathrm{n}(\%)\end{array}$ & $\begin{array}{l}\text { Second } \\
\mathrm{n}(\%)\end{array}$ & $\begin{array}{l}\text { Third } \\
\mathrm{n}(\%)\end{array}$ & \\
\hline Pregnancy test (hCG) & $0(0 \%)$ & $43(14.05 \%)$ & $0(0 \%)$ & $0(0 \%)$ & $0(0 \%)$ & $61(16.62 \%)$ & $0(0 \%)$ & $0(0 \%)$ & $104(4.39 \%)$ \\
\hline $\begin{array}{l}\text { Complete Blood } \\
\text { Count }(\mathrm{CBC})\end{array}$ & $0(0 \%)$ & $95(31.05 \%)$ & $8(2.83 \%)$ & $176(31.43 \%)$ & $0(0 \%)$ & $115(31.34 \%)$ & $5(1.57 \%)$ & $184(34.52 \%)$ & $583(24.61 \%)$ \\
\hline $\begin{array}{l}\text { Blood typing and } \\
\text { antibody screening }\end{array}$ & $0(0 \%)$ & $14(4.58 \%)$ & $0(0 \%)$ & $0(0 \%)$ & $0(0 \%)$ & $11(2.98 \%)$ & $0(0 \%)$ & $0(0 \%)$ & $25(1.06 \%)$ \\
\hline $\begin{array}{l}\text { Glucose fasting plasma, } \\
\text { CUS fasting, Glucose } 2 \mathrm{~h} \\
\text { ABF plasma, CUS } 2 \mathrm{~h} \mathrm{ABF}\end{array}$ & $0(0 \%)$ & $22(7.19 \%)$ & $107(37.81 \%)$ & $185(33.04 \%)$ & $0(0 \%)$ & $18(4.90 \%)$ & $137(43.08 \%)$ & $162(30.39 \%)$ & $631(26.64 \%)$ \\
\hline $\begin{array}{l}\text { Thyroid Stimulating } \\
\text { Hormone (TSH) }\end{array}$ & $0(0 \%)$ & $8(2.61 \%)$ & $17(6.01 \%)$ & $12(2.14 \%)$ & $0(0 \%)$ & $23(6.27 \%)$ & $9(2.83 \%)$ & $13(2.44 \%)$ & $82(3.46 \%)$ \\
\hline Hepatitis B screening & $2(100 \%)$ & $0(0 \%)$ & $0(0 \%)$ & $0(0 \%)$ & $0(0 \%)$ & $1(0.27 \%)$ & $0(0 \%)$ & $0(0 \%)$ & $3(0.13 \%)$ \\
\hline Rubella immunity & $0(0 \%)$ & $0(0 \%)$ & $0(0 \%)$ & $0(0 \%)$ & $0(0 \%)$ & $0(0 \%)$ & $0(0 \%)$ & $0(0 \%)$ & $0(0 \%)$ \\
\hline Urine culture and sensitivity & $0(0 \%)$ & $46(15.03 \%)$ & $27(9.54 \%)$ & $4(0.71 \%)$ & $0(0 \%)$ & $38(10.35 \%)$ & $25(7.86 \%)$ & $7(1.31 \%)$ & $147(6.21 \%)$ \\
\hline Ultrasonography & $0(0 \%)$ & $72(23.53 \%)$ & $124(43.82 \%)$ & $183(32.68 \%)$ & $0(0 \%)$ & $86(23.43 \%)$ & $142(44.65 \%)$ & $167(31.33 \%)$ & $774(32.67 \%)$ \\
\hline STDs & $0(0 \%)$ & $6(1.96 \%)$ & $0(0 \%)$ & $0(0 \%)$ & $0(0 \%)$ & $14(3.81 \%)$ & $0(0 \%)$ & $0(0 \%)$ & $20(0.84 \%)$ \\
\hline Total & $2(100 \%)$ & $306(100 \%)$ & $283(100 \%)$ & $560(100 \%)$ & $0(0 \%)$ & $367(100 \%)$ & $318(100 \%)$ & $533(100 \%)$ & $2369(100 \%)$ \\
\hline
\end{tabular}

Table 4: Pathological data of lactating mother based on symptoms and diagnosis

\begin{tabular}{|c|c|c|c|c|}
\hline \multirow[b]{2}{*}{ Problem } & \multirow[b]{2}{*}{ Symptoms } & $\mathrm{RMCH}$ & \multicolumn{2}{|l|}{ ICMH } \\
\hline & & $\begin{array}{l}\text { Problem responded } \\
\text { lactating mother } \\
\mathrm{n}(\%)\end{array}$ & $\begin{array}{l}\text { Problem responded } \\
\text { lactating mother } \\
\mathrm{n}(\%)\end{array}$ & Total n(\%) \\
\hline Breast engorgement & $\begin{array}{l}\text { Breasts are swollen, firm, painful, shiny } \\
\text { reddish areas, edema and flattened-out nipples }\end{array}$ & $27(25.71 \%)$ & $18(15.79 \%)$ & $45(20.55 \%)$ \\
\hline Sore nipples or breasts & $\begin{array}{l}\text { Erythema, edema, fissures blisters, } \\
\text { white "spots, "yellow or dark } \\
\text { spots and ecchymosis }\end{array}$ & $9(8.57 \%)$ & $13(11.40 \%)$ & $22(10.05 \%)$ \\
\hline Breast abscess & $\begin{array}{l}\text { Painful, swollen lumps, red and warm breast, } \\
\text { fever with high temperature }\end{array}$ & $8(7.62 \%)$ & $5(4.39 \%)$ & $13(5.94 \%)$ \\
\hline Poor milk production & $\begin{array}{l}\text { Infant cries a lot, wants to nurse frequently, } \\
\text { takes very long feedings and does not gain } \\
\text { weight properly }(<20 \mathrm{~g} \text { a day })\end{array}$ & $61(58.10 \%)$ & $78(68.42 \%)$ & $139(63.47 \%)$ \\
\hline Total & & $105(100 \%)$ & $114(100 \%)$ & $219(100 \%)$ \\
\hline
\end{tabular}


Table 5: Distribution of prescribed drugs to pregnant women according to therapeutic classes

\begin{tabular}{|c|c|c|c|c|c|c|c|}
\hline \multirow[b]{3}{*}{ Therapeutic class } & \multicolumn{7}{|c|}{ Gestational age (Trimester) } \\
\hline & \multicolumn{3}{|l|}{$\mathrm{RMCH}$} & \multicolumn{4}{|l|}{ ICMH } \\
\hline & First n(\%) & Second $\mathrm{n}(\%)$ & Third n(\%) & First n(\%) & Second $\mathrm{n}(\%)$ & Third n(\%) & Total n(\%) \\
\hline Anti-infective & $0(0 \%)$ & $25(7.31 \%)$ & $50(10.20 \%)$ & $0(0 \%)$ & $17(4.67 \%)$ & $41(9.09 \%)$ & $133(6.0 \%)$ \\
\hline Anti-ulcerants, anti-emetic & $80(30.19 \%)$ & $38(11.11 \%)$ & $121(24.69 \%)$ & $96(31.58 \%)$ & $63(17.31 \%)$ & $98(21.73 \%)$ & $496(22.38 \%)$ \\
\hline Anti-asthmatic & $0(0 \%)$ & $30(8.77 \%)$ & $0(0 \%)$ & $0(0 \%)$ & $26(7.14 \%)$ & $9(2.0 \%)$ & $65(2.93 \%)$ \\
\hline $\begin{array}{l}\text { Analgesics, antipyretic, } \\
\text { anti-inflammatory }\end{array}$ & $71(26.79 \%)$ & $21(6.14 \%)$ & $29(5.92 \%)$ & $76(25.0 \%)$ & $36(9.89 \%)$ & $31(6.87 \%)$ & $264(11.91 \%)$ \\
\hline $\begin{array}{l}\text { Cardiovascular drugs } \\
\text { including anti-coagulants }\end{array}$ & $0(0 \%)$ & $88(25.73 \%)$ & $0(0 \%)$ & $0(0 \%)$ & $37(10.16 \%)$ & $5(1.11 \%)$ & $130(5.87 \%)$ \\
\hline Dermatological/ anti-fungal & $0(0 \%)$ & $23(6.73 \%)$ & $30(6.12 \%)$ & $0(0 \%)$ & $14(3.85 \%)$ & $22(4.88 \%)$ & $89(4.02 \%)$ \\
\hline Anti-malarial/ anti-protozoal & $0(0 \%)$ & $0(0 \%)$ & $25(5.10 \%)$ & $0(0 \%)$ & $8(2.2 \%)$ & $0(0 \%)$ & $33(1.49 \%)$ \\
\hline Anti-diabetic & $15(5.66 \%)$ & $0(0 \%)$ & $29(5.92 \%)$ & $11(3.62 \%)$ & $6(1.65 \%)$ & $16(3.55 \%)$ & $77(3.47 \%)$ \\
\hline $\begin{array}{l}\text { CNS drugs including } \\
\text { anti-epileptic }\end{array}$ & $0(0 \%)$ & $8(2.34 \%)$ & $0(0 \%)$ & $0(0 \%)$ & $13(3.57 \%)$ & $7(1.55 \%)$ & $28(1.26 \%)$ \\
\hline $\begin{array}{l}\text { Anti-anemic/vitamin } \\
\text { and mineral supplements }\end{array}$ & $95(35.85 \%)$ & $102(29.82 \%)$ & $184(37.55 \%)$ & $115(37.83 \%)$ & $134(36.81 \%)$ & $195(43.24 \%)$ & $825(37.23 \%)$ \\
\hline Hormonal drugs & $4(1.51 \%)$ & $7(2.05 \%)$ & $22(4.49 \%)$ & $6(1.97 \%)$ & $10(2.75 \%)$ & $27(5.99 \%)$ & $76(3.43 \%)$ \\
\hline Total & $265(100 \%)$ & $342(100 \%)$ & $490(100 \%)$ & $304(100 \%)$ & $364(100 \%)$ & $451(100 \%)$ & $2216(100 \%)$ \\
\hline $\begin{array}{l}\text { Mean } \pm \text { SEM of number of drug } \\
\text { prescribed in per prescription }\end{array}$ & & $\begin{array}{l}2.194 \pm 0.025 \\
\mathrm{n}=500\end{array}$ & & & $\begin{array}{l}2.238 \pm 0.028 \\
\mathrm{n}=500\end{array}$ & & $\begin{array}{c}* * 2.216 \pm 0.019 \\
\mathrm{n}=1000\end{array}$ \\
\hline Range of drugs per prescription & & $1-4$ & & & $1-6$ & & $1-6$ \\
\hline
\end{tabular}

$\mathrm{n}=$ Number of pregnant women. ${ }^{* *}$ Differ at the level of 0.50 . Drugs per encounter set by WHO: $1.60-1.80$

Table 6: Distribution of prescribed drugs to pregnant women according to US-FDA categories

\begin{tabular}{|c|c|c|c|c|c|c|c|}
\hline \multirow{3}{*}{$\begin{array}{l}\text { US-FDA } \\
\text { Category }\end{array}$} & \multicolumn{6}{|c|}{ Gestational age (Trimester) } & \multirow[b]{3}{*}{ Total n(\%) } \\
\hline & \multicolumn{3}{|l|}{$\mathrm{RMCH}$} & \multicolumn{3}{|l|}{ ICMH } & \\
\hline & First $n(\%)$ & Second n(\%) & Third n(\%) & First $n(\%)$ & Second n(\%) & Third n(\%) & \\
\hline $\mathrm{A}$ & $135(50.94 \%)$ & $175(51.17 \%)$ & $243(49.59 \%)$ & $168(55.26 \%)$ & $183(50.28 \%)$ & $194(43.02 \%)$ & $1098(49.55 \%)$ \\
\hline B & $79(29.81 \%)$ & $88(25.73 \%)$ & $184(37.55 \%)$ & $74(24.34 \%)$ & $102(28.02 \%)$ & $202(44.79 \%)$ & $729(32.90 \%)$ \\
\hline $\mathrm{C}$ & $51(19.25 \%)$ & $42(12.28 \%)$ & $54(11.02 \%)$ & $62(20.40 \%)$ & $51(14.01 \%)$ & $49(10.86 \%)$ & $309(13.94 \%)$ \\
\hline $\mathrm{D}$ & $0(0 \%)$ & $37(10.82 \%)$ & $9(1.84 \%)$ & $0(0 \%)$ & $28(7.69 \%)$ & $6(1.33 \%)$ & $80(3.61 \%)$ \\
\hline $\mathrm{X}$ & $0(0 \%)$ & $0(0 \%)$ & $0(0 \%)$ & $0(0 \%)$ & $0(0 \%)$ & $0(0 \%)$ & $0(0 \%)$ \\
\hline Total & $265(100 \%)$ & $342(100 \%)$ & $490(100 \%)$ & $304(100 \%)$ & $364(100 \%)$ & $451(100 \%)$ & $2216(100 \%)$ \\
\hline
\end{tabular}

Table 7: Distribution of prescribed drugs to lactating mothers according to therapeutic classes

\begin{tabular}{llll} 
& Lactating mother n(\%) & & \\
& - & ICMH & Total $\mathrm{n}(\%)$ \\
\hline Therapeutic class & RCMH & $172(27.65 \%)$ & $355(29.61 \%)$ \\
Anti-infective & $183(31.72 \%)$ & $102(16.40 \%)$ & $194(16.18 \%)$ \\
Anti-ulcerants, antiemetic & $92(15.94 \%)$ & $35(5.63 \%)$ & $64(5.38 \%)$ \\
Anti-asthmatic & $29(5.03 \%)$ & $84(13.50 \%)$ & $155(12.93 \%)$ \\
Analgesics, antipyretic, anti-inflammatory & $71(12.31 \%)$ & $12(1.93 \%)$ & $20(1.69 \%)$ \\
Cardiovascular drugs including anti-coagulants & $8(1.39 \%)$ & $77(12.38 \%)$ & $158(13.18 \%)$ \\
Dermatological/ antifungal & $81(14.04 \%)$ & $15(2.41 \%)$ & $25(2.09 \%)$ \\
Anti-malarial/ anti-protozoal & $10(1.73 \%)$ & $21(3.38 \%)$ & $38(3.17 \%)$ \\
Anti-diabetic & $17(2.95 \%)$ & $12(1.93 \%)$ & $19(1.58 \%)$ \\
CNS drugs including anti-epileptic & $7(1.21 \%)$ & $81(13.02 \%)$ & $153(12.76 \%)$ \\
Anti-anemic/ Vitamin and mineral supplements & $72(12.48 \%)$ & $11(1.77 \%)$ & $18(1.50 \%)$ \\
Hormonal drugs & $7(1.21 \%)$ & $622(100 \%)$ & $1199(100 \%)$ \\
Total & $577(100 \%)$ & $1.244 \pm 0.024$ & $* * 1.199 \pm 0.014$ \\
Mean \pm SEM of number of drug prescribed in per prescription & $1.154 \pm 0.017$ & $\mathrm{n}=500$ & $\mathrm{n}=1000$ \\
& $\mathrm{n}=500$ & $1-5$ & $1-5$ \\
Range of drugs per prescriptions & $1-3$ & & \\
\hline
\end{tabular}


Table 8: Distribution of prescribed drugs to lactating mother according to Thomas Hale Lactation categories

\begin{tabular}{|c|c|c|c|}
\hline \multirow[b]{2}{*}{ Lactation risk categories } & \multicolumn{2}{|c|}{ Lactating mother $\mathrm{n}(\mathbf{\%})$} & \multirow[b]{2}{*}{ Total n(\%) } \\
\hline & $\mathrm{RMCH}$ & ICMH & \\
\hline L1 & $178(30.85 \%)$ & $216(34.73 \%)$ & $394(32.86 \%)$ \\
\hline $\mathrm{L} 2$ & $297(51.47 \%)$ & $308(49.52 \%)$ & $605(50.46 \%)$ \\
\hline L3 & $93(16.12 \%)$ & $85(13.67 \%)$ & $178(14.85 \%)$ \\
\hline L4 & $9(1.56 \%)$ & $13(2.09 \%)$ & $22(1.83 \%)$ \\
\hline L5 & $0(0 \%)$ & $0(0 \%)$ & $0(0 \%)$ \\
\hline Total & $577(100 \%)$ & $622(100 \%)$ & $1199(100 \%)$ \\
\hline
\end{tabular}

$\mathrm{n}=$ Number of lactating mother

Table 9: Distribution of teratogenic drugs prescribed to pregnant women

\begin{tabular}{llll} 
Distribution of & $\mathrm{n}$ & & \\
teratogenic drugs & RMCH & ICMH & Total (n) \\
\hline Lisinopril & 2 & 1 & 3 \\
Captopril & 1 & 1 & 2 \\
Warfarin & 1 & 2 & 3 \\
Carbamazepine & 1 & 1 & 2 \\
Phenytoin & 1 & 0 & 1 \\
Sodium valporate & 2 & 1 & 3 \\
Carbimazole & 1 & 1 & 2 \\
Total & 9 & 7 & 16
\end{tabular}

Table 10: Distribution of milk inducing and suppressing drugs prescribed to lactating mothers

\begin{tabular}{lrrr}
\hline & $n$ & & \\
& ----------- & \\
Prescribed drugs & RMCH & ICMH & Total n \\
\hline Prescribed milk inducing drugs & & & \\
Domperidone & 33 & 41 & 74 \\
Metoclopramide & 25 & 38 & 63 \\
Sulpiride & 7 & 6 & 13 \\
Chlorpromazine & 4 & 2 & 6 \\
Total & 69 & 87 & 156 \\
Prescribed lactation & & & \\
suppressing drugs & $\mathrm{n}$ & $\mathrm{n}$ & Total n \\
Estrogen & 3 & 2 & 5 \\
Carbergoline & 2 & 0 & 2 \\
Pseudoephedrine & 4 & 7 & 11 \\
Diuretics & 7 & 5 & 12 \\
Bromocriptine & 1 & 0 & 1 \\
Total & 17 & 14 & 31 \\
\hline
\end{tabular}

On the other hand, the therapeutical classes of drugs those were prescribed to lactating mothers also scrutinized to determine the extent of prescription. As shown in Table 7, anti-infective drugs (29.61\%) were the most frequently dispensed drugs at both health care canters followed anti-ulcerants and antiemetic $(16.18 \%)$, dermatological/antifungal $\quad(13.18 \%)$, analgesics, antipyretic and anti-inflammatory (12.93\%), Anti-anemic drugs including vitamin and mineral supplements (12.76\%), anti-asthmatic (5.38\%), antidiabetic (3.17\%), anti-malarial/anti-protozoal (2.09\%), cardiovascular drugs including anti-coagulants (1.69\%), CNS drugs including anti-epileptic (1.58\%), hormonal products $(1.50 \%)$,. On the contrary, according to "Thomas Hale Lactation Risk Categories", 394 women (32.86\%) received a drug from category $\mathrm{L}_{1} ; 605$ women $(50.46 \%)$ received a drug from category $\mathrm{L}_{2} ; 178$ women received a drug from category $\mathrm{L}_{3}(14.85 \%)$; 22 women $(1.83 \%)$ received a drug from category $\mathrm{L}_{4}$ and no lactating mothers were prescribed a drug from category $\mathrm{L}_{5}$ (0\%) (Table 8).

Further analysis was performed to identify the teratogenic dugs of given prescriptions. In this study, 1000 pregnant women and 1000 lactating mothers were included from both hospitals. However, among 1000 pregnant women, 16 women $(1.6 \%)$ were prescribed the drugs of teratogen. Lisinopril, warfarin and sodium valporate were the most frequently prescribed $(n=3)$ among the teratogenic drugs followed by carbamazepine, carbimazole and captopril $(\mathrm{n}=2)$ (Table 9). Also, Table 10 depicts the distribution of both milk inducing drugs and lactation suppressing drugs. It is seen that domperidone was prescribed maximum time $(n=74)$ to increase milk production of breastfeeding mothers at two hospitals and in total 156 breastfeeding mothers $(15.6 \%)$ were received milk inducing drugs from health centers. On the contrary, 31 lactating mothers $(3.1 \%)$ were prescribed the drugs of milk suppressants and among milk suppressants, diuretics $(n=12)$ were included in more prescriptions.

\section{Discussion}

This is the first one conducted at secondary and tertiary care hospitals which demonstrates using of prescribed drugs during pregnancy and lactation in Bangladesh. Similar studies were conducted by Uchenna et al. (2007), Sharma et al. (2006) and Rohra et al. (2008). In this study, not only justified therapeutic classes of drugs but also scrutinized both US Food and Drug Administration (US-FDA) risk categories for pregnant women and Thomas Hale lactation risk categories for breastfeeding mothers to determine the precision of drugs those were prescribed by the health care providers. This study also determined the extent of prescription of drugs which are considered teratogenic for the fetus. Furthermore, both milk inducing and milk suppressing drugs were analyzed to evaluate prescription patterns in Bangladesh. 
The strength of the present study is that the determination of exposure of drugs was based on physical prescriptions rather than on recall, which may lead to bias or under ascertainment (Rockenbauer et al., 2001). The average numbers of drugs prescribed per encounter in the antenatal clinics differ from the standard set by WHO (APED, 1985). The maximum numbers of drugs in a prescription during pregnancy and lactation were 6 and 5. The high numbers may have resulted from prescribing routine drugs along with other medicines. The high number may also increase the risk of adverse drug interactions (Uchenna et al., 2007).

The medicines were prescribed in brand names and this pattern is common in Bangladesh. This means that prescribers here were not complying fully with WHO recommendation that drugs should be prescribed using their international nonproprietary names. The use of brand names by physicians may relate to factors that include the influence of drug promotional activities, pressures of medical representatives who are the employees of drug-distribution or production companies, act as a source of information for both registered and unregistered physicians, offer financial inducements and many other 'gifts' during their visit to the physicians. Also, physicians may have lack of continuing education on the principles of rational prescribing and nonfamiliarity with generic names.

With regards to pathological data of pregnant women (Table 3), it was seen that Ultrasonography was the most common recommendation of physicians. The recommendation of Ultrasonography by physicians may relate to establish and confirm pregnancy date, to determine the number of fetuses, position of a fetus and examine the placental structures, to observe fetal behavior, activity and fetal growth and also to examine the fetal anatomy for presence of abnormalities. Complete Blood Count (CBC) test is another one which did more pregnant women during pregnancy. It was recommended because of developing anemia during pregnancy. During pregnancy, body produces more blood to support the growth of fetus. If body does not get enough iron or certain other nutrients, body might not be able to produce the amount of red blood cells. As a result, anemia may develop in the body. A number of mothers who visited the hospitals had been suffered of low milk supply. The most common causes of low milk supply are insufficient glandular tissue, hormonal or endocrine problems, using hormonal birth control, taking certain medications or herbs, difficult delivery and life style (Pitman, 2016).

With reference to the drugs utilized in pregnancy, it was found that anti-anemic drugs including vitamins and mineral supplements were the most commonly prescribed drugs and this result being same as that observed in Western Nepal (Das et al., 2003). Paracetamol, NSAIDs, anti-emetics, proton pump inhibitors/H2 blockers, antacids, anti-infective i.e. antibiotics and cardiovascular drugs were the other commonly used drugs. However, vitamins and mineral supplements were taken by less than $45 \%$ of women $(95,102$ and 184 women in the first, second and third trimester at RMCH and 115,134 and 195 women in the first, second and third trimester at ICMH, respectively) in the present study. In a prospective survey in Southwestern Finland (Heikkila et al., 1994), iron and vitamin supplementation were the most frequently used drugs, followed by analgesics, tocolytic agents and drugs for chronic conditions and common pregnancy symptoms. In another study from Australia (Maats and Crowther, $2002)$, folate $(69 \%)$, iron (39\%) and multivitamins (27\%) were the most frequently taken drugs by pregnant women.

Later on, we focused on US Food and Drug Administration (US-FDA) risk classification system to evaluate the prescribing behaviors of the physicians to pregnant women. Majority of the drugs belonged to schedule A, B and C. There are some drugs which are not completely safe but were administered as the benefits outweighed the risks. We also focused our analysis on the drugs which are documented as teratogenic in humans. The proportion of pregnant women who were prescribed potentially teratogenic drugs $(1.6 \%)$ is comparable to the published results of Andrade et al. (2006), who have reported that $1.1 \%$ pregnant population in United States received a teratogenic drug. It reveals that there are certain situations where the doctors are left with no option but to prescribe teratogenic drugs when a suitable replacement is not available and/or when the benefits of the drug to the mother outweigh the possible risk to the fetus. The use of anti-epileptic drugs is a good example in this context (Eadie, 2008). However, it is an alarming trend in Bangladesh that many doctors are prescribing the drugs without knowing their significant toxic effects, drug interaction and contraindications.

It is essential that health professionals know the factors that determine the safety of the drugs used during breastfeeding. Such factors may be related to breast milk, to the mother, to the drug or to the infant (Roberto and Lamounier, 2004). Milk composition is a factor which influences how much of a drug is transferred from plasma to milk (Begg et al., 2002). Factors should also be taken into consideration that reduce the ability of mothers to metabolize or excrete the drug may increase infant exposure to the drug (Howard and Lawrence, 1999). Such cases, special precaution should be taken against prescribing drugs to nursing mothers with liver or kidney diseases due to the elevated levels and increased circulation of the drug in the mother's bloodstream. The age of the infant is another factor, should be considered when prescribes a drug used by a nursing mother. Hale classifies infants, according to their ages, as low-risk (6 to 18 months), moderate-risk (younger than 6 months) and high-risk (preterm, 
newborn, clinically unstable infants and those with poor renal function) for the drug of interest (Hale, 2004).

With regards to Table 7 , it is seen that anti-infective drugs i.e. antibiotics were the most commonly prescribed drugs to lactating mothers and it happened because of having caesarean delivery, breast abscess and sore nipples or breasts. Out of $1000,31.1 \%$ women underwent caesarean section to deliver their babies. As a result, more mothers received anti-infective agents to mitigate the prone of infection. Anti-ulcerants, antiemetic, dermatological and antifungal, analgesics, antipyretic, anti-inflammatory, vitamin and mineral supplements were the other commonly prescribed drugs. Furthermore, we arranged prescribed drugs following Thomas Hale Lactation Risk Categories to assess the prescribing behaviors of the health care professionals to lactating mothers. The majority of the drugs used during lactation in the present study, were from category- $\mathrm{L}_{2}$, followed by category- $\mathrm{L}_{1}$. Drugs in category- $\mathrm{L}_{3}$ and category $-\mathrm{L}_{4}$ were also obtained. This may have been in cases where benefits outweigh the risk. Also, we focused our analysis on the drugs which are documented as milk inducing agents and milk suppressants in breast feeding mothers. Galactogogues or milk inducing drugs may be useful in the following situations: Preterm births, maternal or infant disease, adoption or surrogate pregnancy (Gabay, 2002). Drugs which are well known for suppressing milk production, health professionals should be cautious if any of these drugs has to be actually used and should delay their introduction for as long as possible (weeks or months) (Hale, 2003). The careful indication of maternal treatment and the careful selection of drugs often allow breastfeeding to continue uninterruptedly and safely (Bagatin et al., 2001; Lamounier et al., 2000). However, the advantages of breastfeeding are enormous for the infant, whereas the risks of most medications are minimal; most of the information about drugs during lactation is based on fixed doses and on short-term studies. Breastfeeding should only be interrupted or discouraged if there is ample evidence that the drug used by the mother can be harmful to the infant, or when there is no information about the drug and the drug cannot be replaced with another one that is harmless to the infant (Roberto and Lamounier, 2004).

\section{Conclusion}

The present study reveals that the drug utilization pattern was found to be rational as almost all the drugs prescribed were compatible with both US-FDA categorization and Thomas Hale lactation categorization. Anti-anemic drugs including vitamins and minerals were prescribed to pregnant women topped the list of the prescribed medicines whilst anti-infective drugs captured top list of lactation. It is of paramount importance that pediatricians and health professionals should avoid drugs those are known as teratogenic agents and can reduce milk production.

\section{Acknowledgement}

The experiments were partially accomplished under the Higher Education Quality Enhancement Project (HEQEP) sub project CP: 3260 implemented by the University Grants Commission (UGC) of Bangladesh. Authors are thankful to Professor Dr. Mohammad A. Rashid, faculty of pharmacy, University of Dhaka, Bangladesh for providing questionnaires used in the study.

\section{Funding}

This research received no specific grant from any funding agency in the public, commercial, or not-forprofit sectors.

\section{Authors' Contributions}

Mohammad Mohasin Miah: Conducted the experiment, collected and analyzed data. The first draft of the manuscript was written by Mohasin.

Shakil Ahammad Mridha: Collected and analyzed data. Also collected the inevitable documents to write the draft of the manuscript.

Azad Md. Abu Rayhan: Collected and analyzed data, rechecked the draft of the manuscript.

Afia Ferdous: Supervised and designed the project, cross checked the draft of the manuscript and finally approved for the submission.

\section{Ethics}

The experimental protocols were approved by the Institutional Ethical Review Committee of the Faculty of Pharmacy, Stamford University of Bangladesh. Experiments involved humane, were performed as per the ethical guidelines of the National Institutes of Health (NIH), 1985.

\section{References}

APED, 1985. How to Investigate Drug use in Health Facilities: Selected Drug use Indicators. 1st Edn., World Health Organization, Geneva.

AAPCD, 2001. Transfer of drugs and other chemicals into human milk. Pediatrics, 108: 776-89. PMID: 2359677

Andrade, S.E., M.A. Raebel, A.N. Morse, R.L. Davis and K.A. Chan et al., 2006. Use of prescription medications with a potential for fetal harm among pregnant women. Pharmacoepidemiol. Drug Saf., 15: 546-554. DOI: $10.1002 /$ pds. 1235

Bagatin, A.C., L.M.O. Brito, E.G.C. Doria, J.A. Lamounier and G.O. Vieira et al., 2001. Breastfeeding and Drug use. In: Breast Feeding, Rego, J.D., (Ed.), Rio de Janeiro, Atheneu. 
Bakker, M., J. Jentink, F. Vroom, P.B. Van Den Berg and H.E. De Walle et al., 2006. Drug prescription patterns before, during and after pregnancy for chronic, occasional and pregnancy-related drugs in the Netherlands. BJOG, 113: 559-568. DOI: $10.1111 / \mathrm{j} .1471-0528.2006 .00927 . \mathrm{x}$

Banhidy, F., R.B. Lowry and A.E. Czeizel, 2005. Risk and benefit of drug use during pregnancy. Int. J. Med. Sci., 2: 100-106.

Begg, E.J., S.B. Duffull, L.P. Hackett and K.F. Ilett, 2002. Studying drugs in human milk: Time to unify the approach. J. Hum Lact., 18: 323-32.

Benjamin, D.M., 2003. Reducing medication errors and increasing patient safety: Case studies in clinical pharmacology. J. Clin. Pharmacol., 43: 768-783.

Briggs, G.G., R.K. Freeman and S.J. Yaffe, 1994. Drugs in Pregnancy and Lactation: A Reference Guide to Fetal and Neonatal Risk. 4th Edn., Williams and Wilkins.

Das, B., C. Sarkar, A. Datta and S. Bohra, 2003. A Study of drug use during pregnancy in a Teaching Hospital in Western Nepal. Pharmacoepidemiol. Drug Safety, 12: 221-225.

Deborah, E., M. McCarter and M.S. Spaulding, 2005. Medications in pregnancy and lactation. MCN Am. J. Matern Child Nurs., 30: 10-17. PMID: 15622140

Denise, F., 2006. Social drugs and breastfeeding - Health e-Learning.

Eadie, M.J., 2008. Antiepileptic drugs as human teratogens. Expert Opin. Drug Saf., 7: 195-209. DOI: 10.1517/14740338.7.2.195

Gabay, M.P., 2002. Galatogogues: Medications that induce lactation. J. Hum Lact., 18: 274-9.

Hale, T.W., 2003. Medications in breastfeeding mothers of preterm infants. Pediatr Ann., 32: 337-47.

Hale, T.W., 2004. Drug therapy and breastfeeding: pharmacokinetics, risk factors and effects on milk production. Neoreviews.

Heikkila, A.M., R.U. Erkkola and S.E. Nummi, 1994. Use of medication during pregnancy-a prospective cohort study on use and policy of prescribing. Ann. Chir Gynaecol. Suppl., 208: 80-83.

Howard, C.R. and R.A. Lawrence, 1999. Drugs and breastfeeding. Clin. Perinatol., 26: 447-78.

Kacew, S., 1994. Fetal consequences and risks attributed to the use of prescribed and Over-The-Counter (OTC) preparations during pregnancy. Int. J. Clin. Pharmacol. Ther., 32: 335-243.
Lamounier, J.A., E.G.C. Doria, A.C. Bagatin, G.O. Vieira and V.M.B. Serva et al., 2000. Medications and breastfeeding. Medical J. Minas Gerais, 10: 101-11.

Maats, F.H. and C.A. Crowther, 2002. Patterns of vitamin, mineral and herbal supplement use prior to and during pregnancy. Aust. N. Z. J. Obstet Gynaecol., 42: 494-496.

Merck, 2007. Drug use during pregnancy. MMHE, 22: 259-259.

Noha, M.Z. and A.A. Ahmed, 2014. Use, attitudes and knowledge of medications among pregnant women: A Saudi study. Saudi Pharm. J., 22: 419-428. DOI: $10.1016 /$ j.jsps.2013.09.001

Pramanik, D.R. and J.C. Saha, 2010. Drugs in pregnancy. Faridpur Medical Coll. J., 5: 29-31.

Roberto, G.C. and J.A. Lamounier, 2004. Breastfeeding and maternal medications. J. de Pediatria. J. Pediatr. DOI: 10.1590/S00 21-75572004000700011

Rockenbauer, M., J. Olsen, A.E. Czeizel, L. Pedersen and H.T. Sørensen, 2001. Recall bias in a casecontrol surveillance system on the use of medicine during pregnancy. Epidemiology, 12: 461-466. DOI: 10.1097/00001648- 200107000-00017

Rohra, D.K., N. Das, S.I. Azam, N.A. Solangi and Z. Memon et al., 2008. Drug-prescribing patterns during pregnancy in the tertiary care hospitals of Pakistan: A cross sectional study. BMC Pregnancy Childbirth, 8: 24-24. DOI: 10.1186/1471-2393-8-24

Jankowski, R.A.L., 2013. Prescribing for pregnant or lactating women.

Sharma, R., B. Kapoor and U. Verma, 2006. Drug utilization pattern during pregnancy in North India. Indian J. Med. Sci., 60: 277-287.

Sharon, G., 2001. Drug information pharmacist, Christchurch hospital and Evan Begg. Clin.

Pitman, T., 2016. 10 reasons for low milk supply when breastfeeding.

Hale, T.W. and H.E. Rowe, 2014. Medications and Mothers' Milk. 16th Edn., Hale Publishing, Plano, ISBN-10: 1939847389, pp: 1275.

Uchenna, I.E., E.E. Adego, C.O. Azuka and F.E. Ehijie, 2007. Assessment of prescription profile of pregnant women visiting antenatal clinics. Pharmacy Practice, 5: $135-9$. 